\title{
$\frac{1}{\text { inter.noise } 2021}$ \\ 1-5 August | Washington, DC
}

\section{Finite Element Method and Dynamical Energy Analysis in Vibro- acoustics - A Comparative Study}

\author{
Sebastian F. Zettel ${ }^{1}$ \\ German Aerospace Center (DLR) \\ Institute of Aeroelasticity \\ Bunsenstraße 10, 37073 Göttingen \\ Marc Böswald ${ }^{4}$ \\ German Aerospace Center (DLR) \\ Institute of Aeroelasticity \\ Bunsenstraße 10, 37073 Göttingen
}

\author{
René Winter ${ }^{2}$ \\ German Aerospace Center (DLR) \\ Institute of Aeroelasticity \\ Bunsenstraße 10, 37073 Göttingen \\ Martin Richter ${ }^{5}$ \\ University of Nottingham \\ School of Mathematical Sciences \\ University Park, Nottingham
}

\author{
Marco Norambuena ${ }^{3}$ \\ German Aerospace Center (DLR) \\ Institute of Aeroelasticity \\ Bunsenstraße 10, 37073 Göttingen \\ Gregor Tanner ${ }^{6}$ \\ University of Nottingham \\ School of Mathematical Sciences \\ University Park, Nottingham
}

\begin{abstract}
Future aircraft concepts utilizing innovative lightweight structures and novel propulsion concepts are a necessity for long term sustainable air travel. While the development is mainly driven by lightweight design and fuel-efficient propulsion, these concepts may pose new challenges for the vibroacoustic analysis of cabin structures and the associated noise impact on passengers. Finite Element (FE) models derived from aircraft pre-design data are not optimized for use in acoustic analyses, i.e. the mesh typically is too coarse to provide meaningful results. At the same time, setting up Statistical Energy Analysis models for this specific purpose is adding another time-consuming step. An alternative possibly exists in the Dynamical Energy Analysis (DEA). This method allows to calculate the acoustic behavior of thin-walled structures in higher frequency ranges simply using existing $F E$ meshes. In this paper an experimental lightweight aluminum structure and its respective FE model is investigated for a frequency range up to $5000 \mathrm{~Hz}$. A comparison in terms of vibrational energy between DEA, FEM and measurement results is presented. Finally, a lower-bound frequency range is identified in which DEA and FEM correlate and thus allow a substitution for further simulations at higher frequencies. To ensure the transferability of the results to structures of other dimensions and materials, frequency scale factors based on analytical equations are derived.
\end{abstract}

\footnotetext{
${ }^{1}$ Sebastian.Zettel@dlr.de

${ }^{2}$ Rene.Winter@dlr.de

${ }^{3}$ Marco.Norambuena@dlr.de

${ }^{4}$ Marc.Boeswald@dlr.de

${ }^{5}$ Martin.Richter@nottingham.ac.uk

${ }^{6}$ Gregor.Tanner@nottingham.ac.uk
} 


\section{INTRODUCTION}

Vibroacoustic analyses mainly take place in the mid- and in the high-frequency range. While it is possible to simulate the responses of structures using the Finite Element Method (FEM), the high modal densities and accompanying modal overlap at higher frequencies require the FE meshes to have a high resolution to assess the dominant wavelengths in the structure [1]. This of course comes with high calculation costs. Additionally, most of the time a refinement of FE meshes is not trivial especially due to the fact that existing meshes in the design stage are primarily developed for global load-case analyses of the aircrafts structure and not for means of vibro-acoustics.

An alternative approach for high frequencies is the so-called Statistical Energy Analysis (SEA). It assumes that certain parts or components of a structure are considered as energy storage, e.g. for the kinetic energy of the vibration [2]. Only the total amount of energy is considered for a specific component but not the spatial distribution within the component. Most complex structures are assembled of multiple components which store different amounts of energy respectively. The coupling of the components will lead to energy transport from one component to the other until a global balance in the energy distribution is reached. Identifying those components is not always a straight forward task especially for larger structures. Especially, the identification of the necessary coupling loss-factors for the analysis adds another time-consuming and difficult step.

Another promising approach and the one used in this paper, is Dynamical Energy Analysis (DEA). It is a ray-tracing based method [3]. Such methods apply a high-frequency asymptotic to the vibrational equations to get to a formulation in terms of an Eikonal equation (ray-theoretical approximation to the scalar wave equation). This follows single trajectories of vibrational excitation through the complex domains [4]. DEA aims to be an almost black-box computation for the high-frequency range. One of the main advantages of DEA is the relative irrelevance of the size of the mesh discretization. Even for results at high frequencies, it is only necessary to assert that the wavelengths of pressure, shear, and bending waves are small compared to the structures encountered. The mesh has to resolve these geometrically relevant structures but can be arbitrarily coarse beyond that. This allows the direct use of coarse FE meshes from the aircraft's design phase to assess the vibro-acoustic behavior utilizing the DEA method.

Studies have shown that DEA provides sufficiently accurate frequency response results for simple and complex structures [5, 6]. However, there has not been any focus on the applicable frequency range especially with focus on the lower frequency limit. Generally, DEA works more accurately the higher the observed frequencies are. Additionally, as already stated above, the dominant wavelengths should be shorter than the encountered components of the structure. At high frequencies these premises are satisfied. However, for the low-frequency range structural responses feature global deformation. In the mid-frequency range the global and local behavior of the plate cannot be separated properly because low-frequency and high-frequency components (and thus shorter and longer wavelengths) are present simultaneously [7]. Due to the peculiarities of the mid-frequency range the DEA might still be able to provide sufficient frequency responses results for this range at least partially. This would allow to identify lower frequency bounds for an application of the DEA and provide knowledge regarding a possible transition range from FEM to DEA in order to use these two methods complementarily in the future.

In this paper a laboratory structure consisting of a rib-stiffened plate made of aluminum is investigated. The vibration transport behavior of this rib-stiffened plate features similar effects as an aircraft fuselage assembled of frames, stringers and skin. In order to exclude undesired damping effects, the structure was milled from a solid piece of aluminum. The response of the structure due to single-point excitation with a shaker was measured by a Laser Doppler Vibrometer (LDV). Corresponding FE models representing the structure were used for FEM and DEA computations.

In order to assess the correlation of the FEM and DEA results in comparison to measurements of the laboratory structure a criterion which delivers consistent results over the full frequency range from low to high frequencies is necessary. Here the Energy Correlation Criterion (ECC) will be used [7]. 
A commonly used correlation criterion is the Modal Assurance Criterion (MAC). It utilizes modal parameters and is only applicable for low frequencies where experimental modal analysis can be applied. The Frequency Response Assurance Criterion works based on spectral response data but is highly prone to errors due to a high sensitivity to uncertainties in the structure and measurements [9, 10]. The ECC uses an approach based on the spatial distribution of kinetic energy in discrete frequency bands. This enables the ECC to deliver consistent results over a wide frequency range from low to high frequencies. At the same time the ECC is robust against errors arising from uncertainties in the structure and the measurement.

In the part Methods and Experiments of this paper the used laboratory structure, the corresponding FE models and the ECC will be described in more detail. For further information regarding the DEA reference to the mentioned publications is made. In the results part a correlation study using the energy correlation criterion is conducted. Afterwards the transferability of results is ensured by deriving frequency scale factors based on analytic equations for structures with other dimensions and material.

\section{METHODS AND EXPERIMENTS}

To address the task of identifying a lower frequency bound for DEA analyses in order to be able to use FEM and DEA complementarily for full frequency range assessments, an approach based on correlating experimental and simulation data is used. The datasets will be generated using an experimental structure and its virtual representation in form of FE meshes. The experimental data set will act as reference because it represents the real-world behavior of the structure and data can be acquired for the observed frequency range of $50-5000 \mathrm{~Hz}$ in a high quality by using an LDV. For FEM and DEA respectively, different FE meshes will be used. A high-density model was used for the FEM computations. It was previously experimentally validated using EMA (Experimental Modal Analysis). For the DEA a coarse FE mesh will be used which represents a mesh in the design phase. The ECC will be the center piece of this paper to provide the correlation of the data sets for the observed frequency range. This will provide insights on how well the different simulation methods work in specific frequency ranges correlate and allows us to identify transition ranges for future analyses.

\subsection{Experimental setup}

The investigated laboratory structure is a stiffened aluminum plate and was designed with the intent to show the same vibro-acoustic effects as real-world lightweight structures do, while being optimized for experimental usage to eliminate as much structural uncertainties as possible, see Figure 1. The plate was manufactured from a monolithic aluminum plate with dimensions of $0.8 \mathrm{~m} \times 1 \mathrm{~m} \mathrm{x}$ $0.03 \mathrm{~m}$. The stiffened structure was manufactured by CNC milling to ensure that no joints of any kind exist to eliminate friction. The design of the structure was focused on avoiding symmetries to create distinct vibrational transfer paths. The vertical running stiffeners have a height of $1.1 \mathrm{~cm}$ while the horizontal stiffeners are $0.6 \mathrm{~cm}$ high. All skin fields have thicknesses of $1 \mathrm{~mm}$. The material properties of the plate are as follows [11]: Young's modulus $E=70 \mathrm{GPa}$, density $\rho=2660 \mathrm{~kg} / \mathrm{m}^{3}$, Poisson ratio $v=0.34$. The material inherent loss-factor for aluminum was assumed to be $\eta=0.005$.

The experimental setups consist of the plate attached at four points to a bungee-string suspension, providing an almost free-free boundary condition, see Figure $1 \mathrm{~b}$. The normal velocity field of the plate was measured from the unmilled surface of the plate using an LDV attached to a scanning unit developed at DLR [12]. The scanning unit allows to perform automated measurements of the plate. Only the plate's inner region where stiffener and skin fields are present has been measured. The outer surrounding thicker frame to which the suspension is attached to was left out.

The plate is excited by a shaker attached to one of the stiffener crossings, see Figure 1a, using a random signal. The used shaker is limited to a frequency range of $50-5000 \mathrm{~Hz}$ which explains the observed range stated earlier. The velocity field is measured at 19200 equally spaced measurement points on the flat backside of the plate with a sample rate of $10240 \mathrm{~Hz}$. The measured velocity field in time-domain was transformed to the frequency-domain using Welch's method resulting in $5 \mathrm{~Hz}$ 
frequency resolution. Only the plate's inner region where stiffener and skin fields are present has been measured. The outer surrounding thicker frame to which the suspension is attached to was left out.
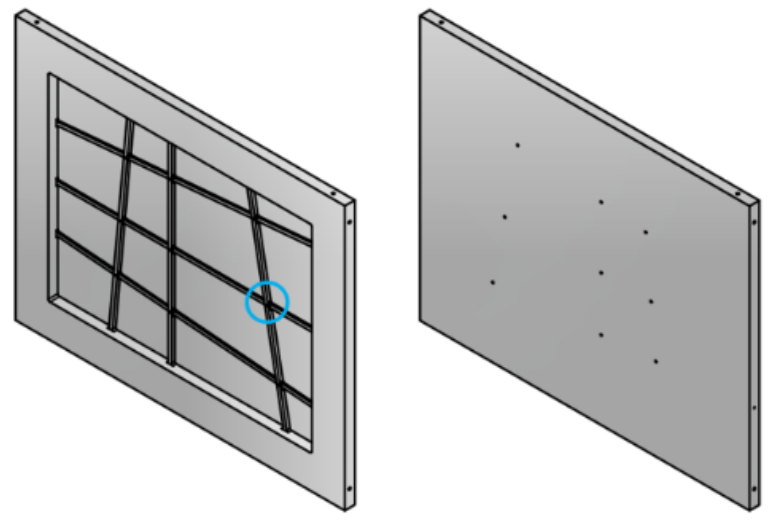

(a)

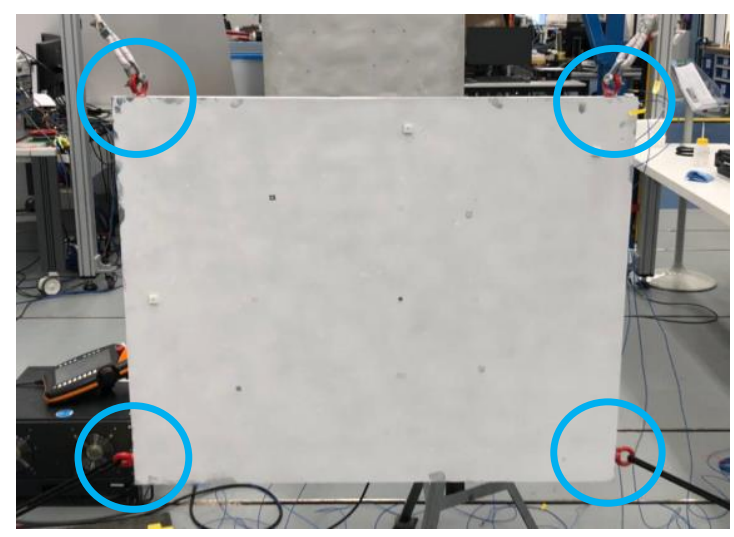

(b)

Figure 1: (a) Plate structure (front and back in isometric view) with excitation point marked [11], (b) Plate suspended by bungee-strings with marked attachment locations (backside view) [11]

\subsection{Energy Correlation Criterion}

The ECC is an energy-based correlation criterion. It utilizes the total kinetic energy of a discrete number of specified subcomponents of a structure similar to the SEA and computes a correlation value using a vector-correlation. The following short summary of the ECC was written based on the publication of Biedermann et. al [8].

The kinetic energy of a point-mass performing a harmonic oscillation is described by Equation 1:

$$
E_{k i n}(\Omega)=\frac{1}{2} m \widehat{v}^{2}(\Omega)
$$

with $\widehat{v}(\Omega)$ being the absolute frequency dependent velocity of a degree-of-freedom (DOF) normal to the structures surface and $m$ being the mass. The frequency dependent velocity can be determined easily in simulations using the FEM and experiments by measuring the velocity field, even for high frequencies where high modal density and overlap is existent.

To calculate the total kinetic energy for experiment or simulation $E_{\text {kin }}^{\text {exp }}$ of a subcomponent $i$ of the structure in a frequency-band $n$ the discrete kinetic energies in the substructure are summarized along a discrete number of spectral lines $l=1, \ldots, L$. For discrete numbers of DOFs from measurement $(\exp ) k=1, \ldots, K$ and simulation $($ num $) \mathrm{o}=1, \ldots, O$ the total kinetic energies result in Equation 2 and Equation 3:

$$
\begin{aligned}
& E_{\text {kin }}^{\text {exp }}(i, n)=\frac{1}{2} \sum_{l=1}^{L} \sum_{k=1}^{K} m_{k}^{\text {exp }}\left(\hat{v}_{k}^{\text {exp }}\left(\Omega_{l}\right)\right)^{2} \Delta \Omega \text { and } \\
& E_{\text {kin }}^{\text {num }}(i, n)=\frac{1}{2} \sum_{l=1}^{L} \sum_{o=1}^{O} m_{o}^{\text {num }}\left(\hat{v}_{o}^{\text {num }}\left(\Omega_{l}\right)\right)^{2} \Delta \Omega
\end{aligned}
$$

with $\Delta \Omega$ describing the chosen frequency-resolution. As can be seen that in each discrete frequency band $\mathrm{n}$, the energy distribution in the structure in described by the vector $E_{\text {kin }}^{\text {exp }}$ num $(n)$ that has as many elements as subcomponents in the structure.

The issue of having meshes with different densities is addressed by the ECC utilizing a mass-condensation. A partial mass is assigned to each of the DOFs of the structure. A finer mesh will have less mass per DOF than a coarser mesh with fewer DOFs. 
The correlation of energies between experiment and simulation is performed by using a vector correlation criterion. The resulting ECC value determines the level of linear dependency between the energy distributions of experiment and simulation. The calculation of the ECC value for the $n$-th frequency band is described by Equation 4:

$$
\operatorname{ECC}(n)=\frac{\left(\left\{E_{\text {kin }}^{\text {exp }}(n)\right\}^{T}\left\{E_{\text {kin }}^{\text {num }}(n)\right\}\right)^{2}}{\left\{E_{\text {kin }}^{\text {exp }}(n)\right\}^{T}\left\{E_{\text {kin }}^{\text {exp }}(n)\right\}\left\{E_{\text {kin }}^{\text {num }}(n)\right\}^{T}\left\{E_{\text {kin }}^{\text {num }}(n)\right\}} \text { with } n=1,2, \ldots, N .
$$

The ECC correlation values lie in a range of 0 to 1 with 0 showing no linear dependency and 1 showing an exact linear dependency. Biedermann et. al [8] describe that values above 0.8 indicate a high level of linear dependency between the energy distributions of experiment and simulation. This limit will be adopted here for the following evaluations.

In this paper the experimental plate described in Chapter 2.1 is subdivided in 49 components (16 skin fields, 9 crossings, 12 high stiffeners (ribs), 12 low stiffeners (stringers)) as can be seen in Figure 2. As already stated the frame of the experimental plate is not considered in this analysis. This division of subcomponents is coarse especially in case for the skin fields, where each one acts as one subcomponent. Coarse subcomponents enforce a stronger spatial averaging during the ECC calculation and eliminates uncertainties in doing so. Thus, for this first investigation it was decided to postpone a further refinement of the main subcomponents to a time where uncertainties regarding the DEA and the chosen models are better known. Regarding the spectral integration an averaging utilizing 1/3octave bands were used.

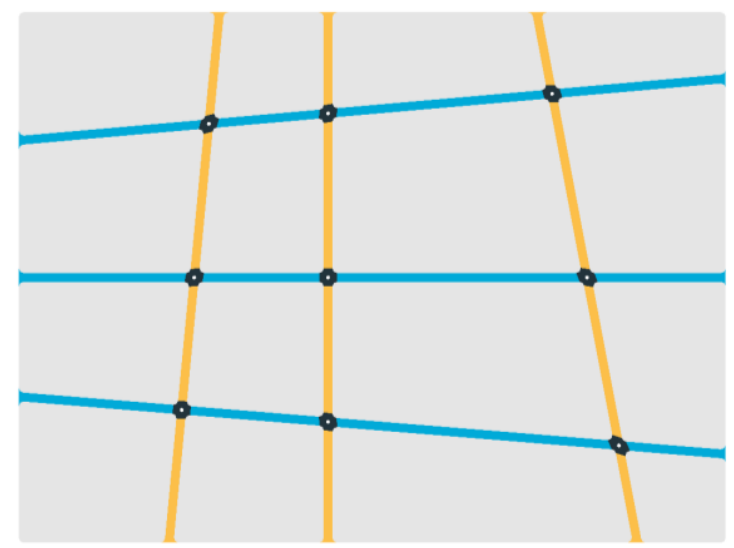

Figure 2: Allocation of the subcomponents for the experimental plate (grey - skin fields, yellow high stiffeners, black - crossings with height of high stiffeners, blue - low stiffeners)

\subsection{FEM and DEA simulation setup}

Simulations using FEM and DEA were performed. The development of the model for FEM simulations showed that volume elements are necessary in order to represent the behavior of the experimental structure properly. However, the current development status of the DEA only allows to use shell elements and these only without applying offsets (existing offsets are neglected). Therefore, two different models were used which will be described in the following. Subsequently, it is shown in a study that the models provide comparable results in the relevant frequency range. It is important to mention that the mentioned limitation does not apply in the modeling of most aeronautical structures.

The model used for FEM simulations (further addressed as model "A") is built-up as a hybrid mesh consisting of shell and volume elements. Due to the complex geometry of the experimental plate, it is divided into two subcomponents which are meshed separately. A thin plate part, spanning the entire backside of the plate and a part containing the stiffeners and the frame, see Figure 3.

The thin plate part is meshed with CQUAD4 elements assigned with a constant thickness of $1 \mathrm{~mm}$, while the volume part is meshed with stacked CHEXA elements modeling the thickness of frame and 

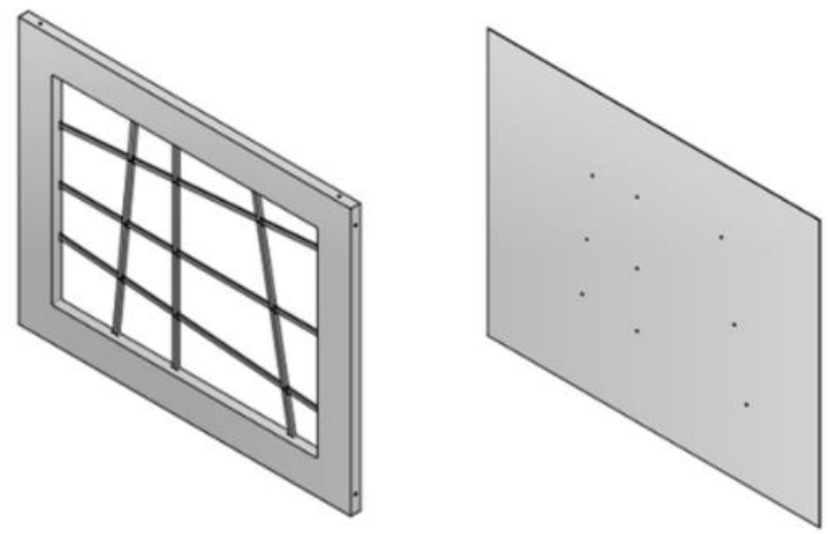

Figure 3: Representation of plate structure divided into volume part (left) and shell part (right) [11] stiffeners. Figure 4 gives a qualitative impression of the hybrid FE mesh and shows the adaption of the mesh to the topology of the experimental structure. The global element edge length was set to 5 $\mathrm{mm}$. Due to the plate's geometry the mesh density varies but the coarsest parts of the mesh, e.g. frame corners, feature $5 \mathrm{~mm}$ element edge lengths. Based on a wavenumber analysis this global element edge length is capable of resolving the dominant waves at the maximum frequency of $5000 \mathrm{~Hz}$.

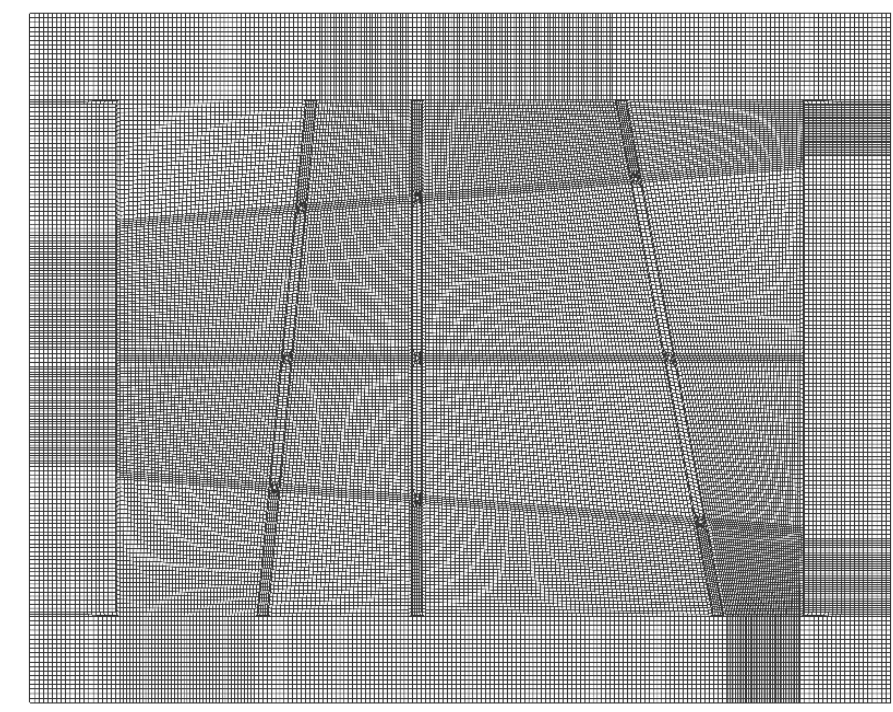

Figure 4: Hybrid FE mesh with 5mm edge length (frontal view)

The hybrid FE model was developed for a model-validation and -updating of the experimental structure for low and high frequencies. It showed superior results compared to a mesh modeled with volume elements only. An EMA was performed up to $400 \mathrm{~Hz}$ and a correlation study using the MAC showed good results between simulation and experiment for the low-frequency range up to $230 \mathrm{~Hz}$. Between $230-300 \mathrm{~Hz}$ linear dependencies decreased due to rising numbers of modes showing vibrations in single skin fields. From $300 \mathrm{~Hz}$ to $400 \mathrm{~Hz}$ linear dependencies increased again [11].

The velocity field of the plate was calculated with Nastran's Solution 111 and a point force excitation at the same location specified as in Figure 1a. Results with a frequency step size of $5 \mathrm{~Hz}$ were requested. Only the velocities of the hybrid FE mesh's DOF on the shell part of the model were used for the ECC calculation, still considering the respective masses of the stiffeners.

The model for the DEA simulation (further addressed as model "B") has the same mesh layout as the hybrid model but features only CQUAD4 elements and an increased element edge length of $20 \mathrm{~mm}$. This mesh resolution represents a mesh in the design phase. The plate's thicknesses of frame and stiffeners are considered by the element properties. The mesh consists of 3553 nodes and 3438 CQUAD4. During the calculation process of the DEA the CQUAD4 element edges are split into a fixed number of sub-edges and the evolving new mesh grid is re-meshed with CTRIA3 elements. 
Here a sub-division of 4 were used to get to the same mesh grid density as the hybrid model. Thus, the internally used DEA mesh consist of a grid of 55465 nodes and 110016 elements. The DEA provides kinetic energies results for every DOF directly.

The DEA simulation was performed for $50-5000 \mathrm{~Hz}$ with a frequency step size of $5 \mathrm{~Hz}$. This $5 \mathrm{~Hz}$ frequency step size is also the limiting factor for the step sizes of FEM and measurement. For such a big frequency range the calculation of DEA results is very time consuming ( $\sim 3$ minutes per frequency for the used model) and thus was limited to the $5 \mathrm{~Hz}$ steps. This was also an advantage for the measurement as the time per point could be reduced to 5 seconds which was enough to generate sufficient spectral estimates for the resulting $5 \mathrm{~Hz}$ bin averages.

In the DEA simulation the excitation is not provided by a point force but an initial energy density. Here an initial energy density of $e=1 \mathrm{~J} / \mathrm{m}^{2}$ was used. Currently a formula to relate initial energy densities to an equal point force is only available for excitations on a simple plate without nearby boundaries. Therefore, a corresponding excitation of the experimental structure would only have been possible in the center of one of the skin fields. In terms of the excitation during the experiment this would not have been reasonable due to a lack of energy input into the structure. However, the DEA and FEM calculation assumes linear behavior of the models making the results qualitatively comparable.

The comparability of the two models was verified applying the ECC to two datasets generated by FEM simulations. This step is important as it shows if model B provides relevant results in the highfrequency range where the DEA operates best even though the models have structural differences. For the computation of the two datasets, model A and a new model $\mathrm{C}$ which has the exact same builtup as model B but with an element edge length of $5 \mathrm{~mm}$ was used. The creation of model $\mathrm{C}$ was necessary because model B would have been to coarse for a direct comparison of FEM results. Figure 5 shows the results of the ECC evaluation. The result plot shows the ECC values over frequency (1/3octave bands). The ECC limit at 0.8 specifies the lower limit for identifying ECC results which show a high linear dependency between the datasets.

At low frequencies the models show a high linear dependency which can also be confirmed by the first eigenfrequencies being $70 \mathrm{~Hz}$ for model $\mathrm{A}$ and $68 \mathrm{~Hz}$ for model $\mathrm{C}$. In the mid-frequency range the ECC values are far below the 0.8 limit but increase with higher frequencies. From $500 \mathrm{~Hz}$ on, the ECC values are above or close to 0.8 and indicate a high similarity of the energy distributions in the models. These results show that the models behave similar especially in the high-frequency range regardless of their structural differences.

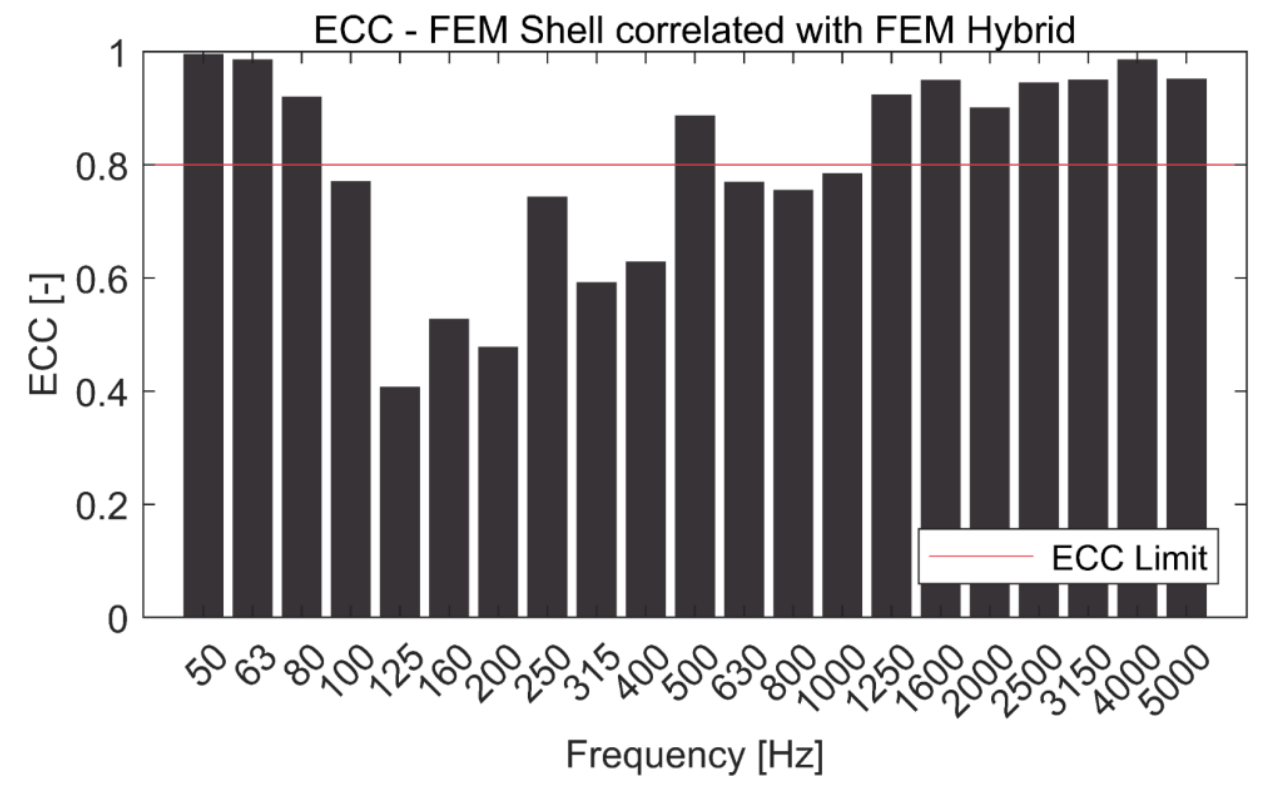

Figure 5: ECC evaluation between FEM shell model C and FEM hybrid model A 


\section{CORRELATION STUDY USING THE ENERGY CORRELATION CRITERION}

In the following, two ECC evaluations will be presented. First, the comparison of FEM and DEA simulation with the measurement data. This evaluation will show if the models are capable of providing sufficient results regarding the structural behavior of the plate, especially at high frequencies which are in focus here. Second, a direct comparison of FEM and DEA simulation which will enable to identify a lower frequency bound for an application of the DEA instead of FEM for high frequencies.

Figure 6 shows the FEM and DEA results in comparison to the measurement data. As expected the ECC values are very high (> 0.98) for low frequencies for the FE hybrid model. This high level of linear dependency results from the previous model updating of the hybrid model. The frequencyrange from $160-315 \mathrm{~Hz}$ is rather volatile with ECC values mostly below the limit of 0.8 . However, from $400 \mathrm{~Hz}$ upwards the ECC values are either close to 0.8 or well above (>0.86) the limit except for the bin at $800 \mathrm{~Hz}$. These results show that the FEM model depicts the behavior of the experimental structure well, especially at low and high frequencies.

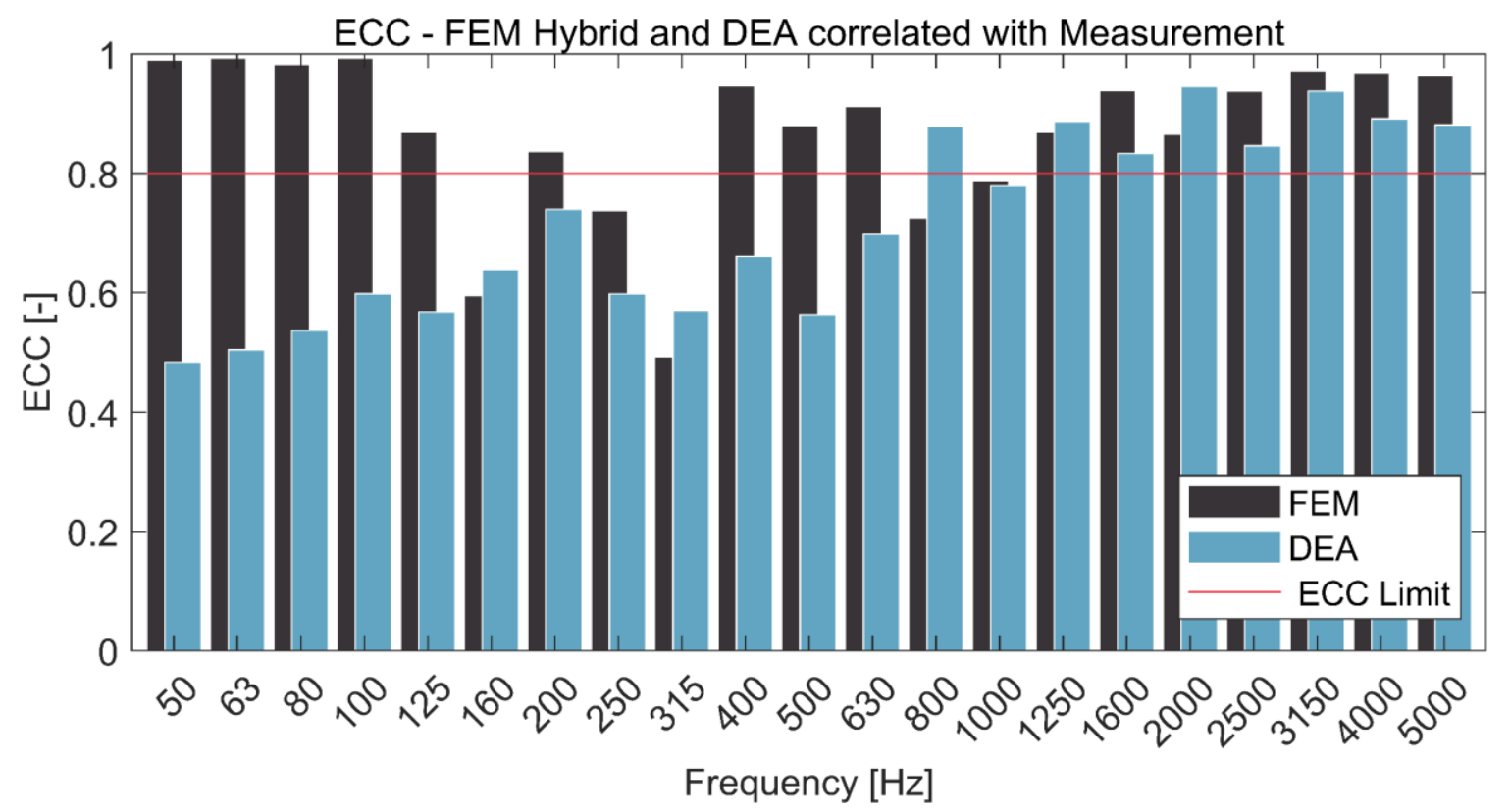

Figure 6: ECC evaluation for the DEA and FEM Hybrid model compared to measurement data

The evaluation for the DEA simulation shows, as expected, low linear dependencies for low frequencies but an almost linear increase of ECC values towards higher frequencies. From $800 \mathrm{~Hz}$ upwards the ECC values are either well above the limit or close to it. As for the FEM model it can be stated that the DEA represents the behavior of the experimental structure very well in the high-frequency range as it is supposed to.

In Figure 7 the direct comparison of the DEA model and the FEM hybrid model is presented. The linear dependencies are low for the low-frequency range due to the DEA. But with increasing frequencies the ECC values get closer to 0.8 almost linear as was already the case in Figure 6. From $1000 \mathrm{~Hz}$ upwards the linear dependency is well above the 0.8 limit and indicates similar behavior of the two models.

The presented results show that a transition from a FEM analysis to an analysis using DEA is possible in a frequency range of about $800-1000 \mathrm{~Hz}$ for the structure used in this paper. Even though the ECC values for the correlation of the DEA model with the FE hybrid model are above 0.8 only starting from $1000 \mathrm{~Hz}$, the two bands at $630 \mathrm{~Hz}$ and $800 \mathrm{~Hz}$ are close to the limit. Additionally, the correlation of the DEA model with measurement data in Figure 6 shows that the ECC values are above 0.8 at $800 \mathrm{~Hz}$ already. 


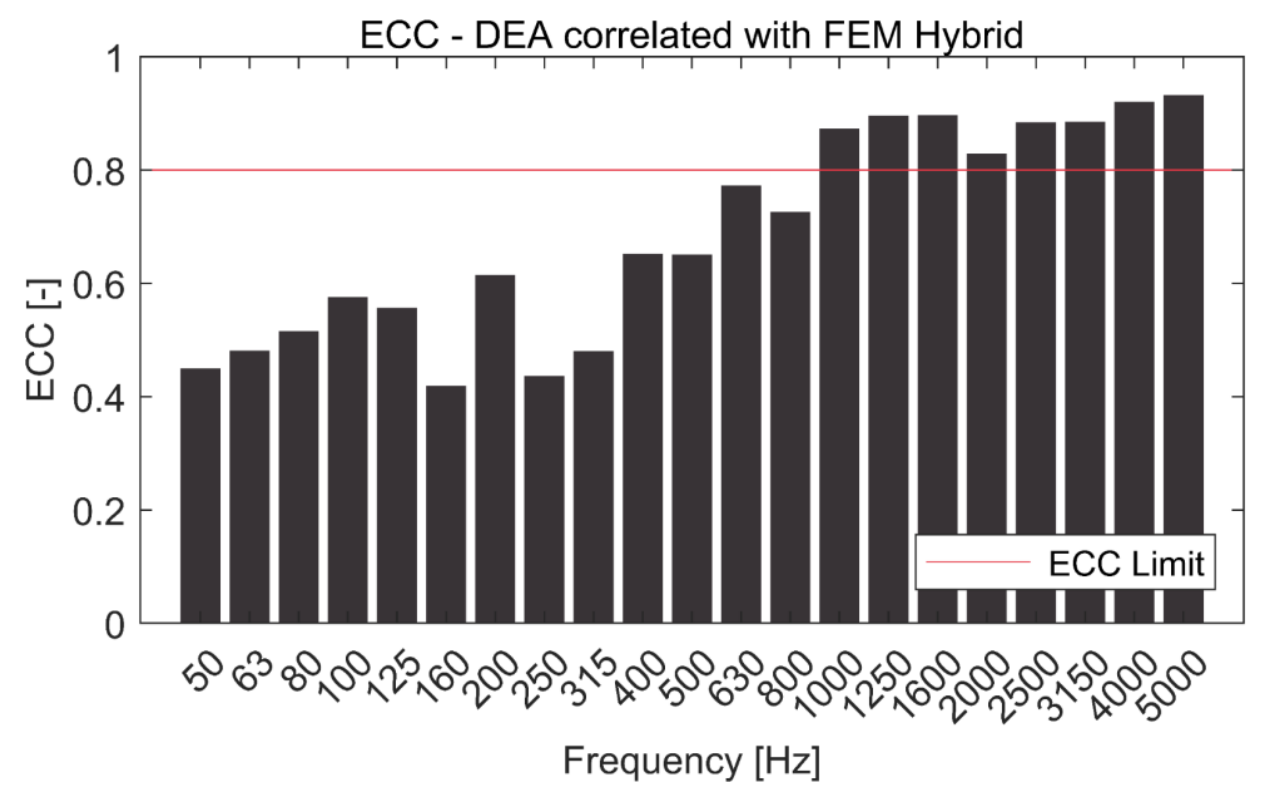

Figure 7: ECC evaluation between the DEA and FEM Hybrid model

\section{TRANSFERABILITY OF RESULTS TO STRUCTURES OF OTHER DIMENSIONS}

The results presented in Chapter 3 are only valid for the experimental structure used in this paper. In order to transfer the findings of this paper to plate-like structures with other dimensions, a rule-of-thumb is derived from analytical equations assuming isotropic material. The lower frequency limit for using DEA cannot be specified to an exact frequency and actually, the frequency limit is not needed to be known precisely. However, the derived results of this chapter will provide a rough estimate on how the lower frequency bound change when plates with other dimensions are assessed. For the following derivation it is assumed that the lower frequency limit will change just in the same way as the eigenfrequencies of a structure when the dimensions change. This is a reasonable assumption, because in SEA the lower frequency limit is also related to the eigenfrequencies and in particular to the number of eigenfrequencies in a frequency band.

The eigenfrequencies of a rectangular plate with all edges simply-supported and constant thickness $h$ can be calculated with Equation 5 [13, p.92]:

$$
\omega_{m n}=\pi^{2}\left[\left(\frac{m}{a}\right)^{2}+\left(\frac{n}{b}\right)^{2}\right] \sqrt{\frac{D}{\rho h}}
$$

with the number of the eigen-modes $m$ and $n$ along the $\mathrm{x}$ and $\mathrm{y}$ direction of the rectangular plate [13, p.81], the dimensions of the plate $a$ and $b$ in $\mathrm{x}$ and y direction and density $\rho . D$ describes the flexural stiffness of the plate when assuming isotropic material (see Equation 6) with the Poisson's ratio $v$ and Young's Modulus E.

$$
D=\frac{E h^{3}}{12\left(1-v^{2}\right)}
$$

By substituting Equation 6 in Equation 5 the resulting equation can be reduced to an equation which is only proportional to the dimensional coefficients $h, a^{2}$ and $b^{2}$ (see Equation 7).

$$
\omega_{\text {geom }, i} \sim h_{i}\left(\frac{1}{a_{i}^{2}}+\frac{1}{b_{i}^{2}}\right)
$$

Plates of different geometric dimensions will feature different eigenfrequencies. Calculating the ratio of two eigenfrequencies utilizing Equation 7 yields the frequency scale factor (see Equation 8). As the equation contains summations it cannot be simplified further without affecting readability. 


$$
\Delta_{\text {freq }}=\frac{\omega_{\text {geom }, 2}}{\omega_{\text {geom }, 1}}=\frac{h_{2}\left(\frac{1}{a_{2}{ }^{2}}+\frac{1}{b_{2}{ }^{2}}\right)}{h_{1}\left(\frac{1}{a_{1}{ }^{2}}+\frac{1}{b_{1}{ }^{2}}\right)}
$$

The frequency scale factor $\Delta_{\text {freq }}$ can be calculated for a variety of dimension scales $\Delta_{\text {geom }}$ for $h, a$ and/or $b$ and can be plotted as a set of graphs which indicate the eigenfrequency behavior of plates for different cases, see Figure 8. Here geometric scaling in a range of $\Delta_{\text {geom }}=[0.1,10]$ is considered but can be extended arbitrarily. The two graphs ( $a=$ const.) and ( $a, h=$ const.) as listed in the legend, have the exact same progressions as ( $b=$ const.) and ( $b, h=$ const.). Therefore, only one variant is presented in Figure 8.

In the same way as the frequency scale factor was derived for a geometric scaling of plates it is possible to derive frequency scaling factors for the scaling of material properties $\Delta_{\text {mat }}$. For the sake of brevity this derivation is not presented in detail. However, the graphs describing the progression of frequency scale factors for scaling $\mathrm{E}, v$ and $\rho$ are identical to two graphs for geometric scaling (see Figure 8). The material scaling $\Delta_{\text {mat }}$ is considered in the same range as $\Delta_{\text {geom }}$.

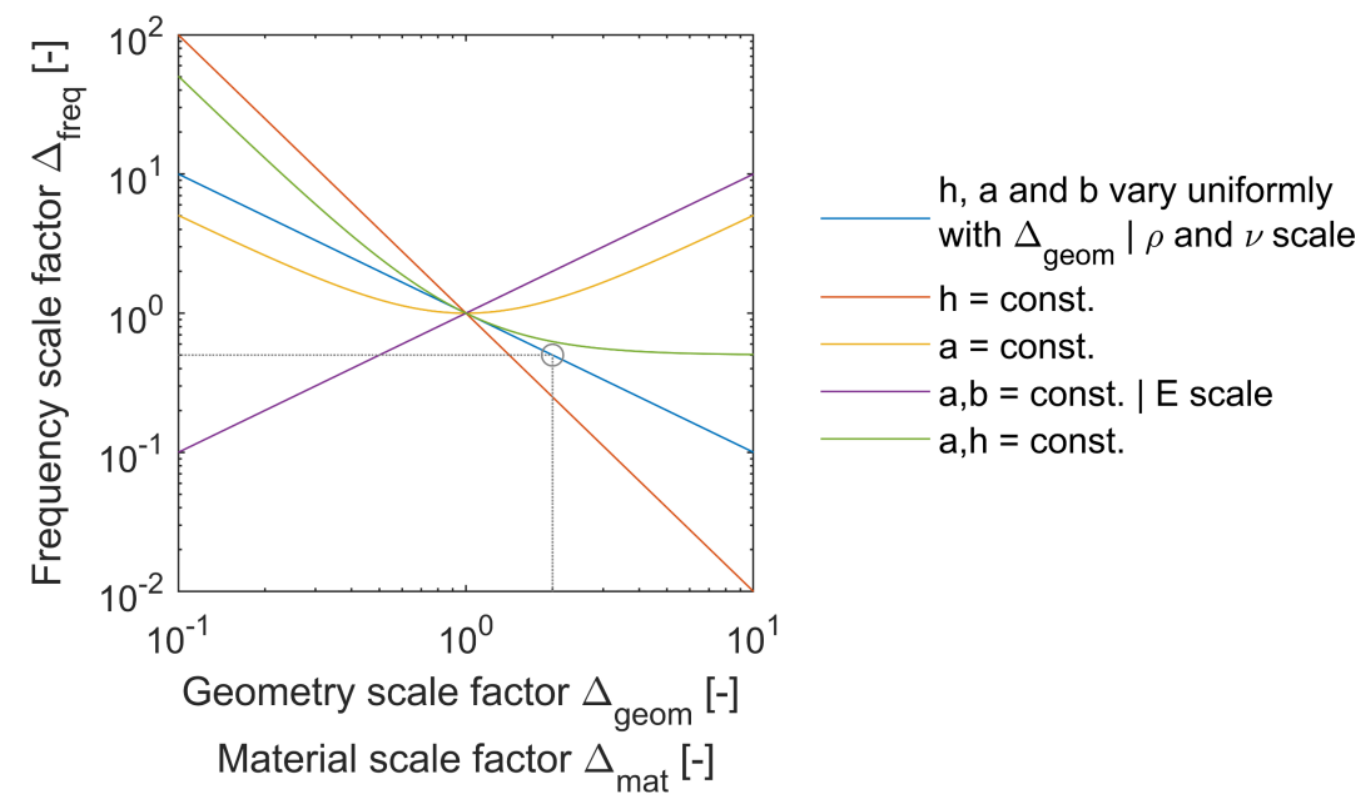

Figure 8: Frequency scale factor in dependence of geometry scale factor for five cases

As an example, we consider the graph which represents the eigenfrequency behavior if all dimensions $(h, a$ and $b)$ are scaled uniformly (i.e. blue line in Figure 8). A doubling of these dimensions $\left(\Delta_{\text {geom }}\right.$ $=2$ ) result in a frequency scale factor of $\Delta_{\text {freq }}=0.5$ (see grey circle). This indicates that all eigenfrequencies of a plate reduce to half of their size when all dimensions are doubled.

If the change of a plate's dimensions cannot be fully accounted for by one of the graphs in Figure 8, a consecutive application of the graphs is valid according to the principle of superposition. For example, if a plates dimension for $a$ and $b$ double but the thickness $h$ only increases $50 \%$ it is valid to determine a frequency scale factor along the ( $h=$ const.)-graph first and afterwards determine another factor along the ( $a, b=$ const.)-graph. The two factors need to be multiplied to determine the overall frequency scale factor for this dimension change.

\section{CONCLUSION}

The aim of this paper was to identify a lower frequency bound from which it is able to transition from FEM analyses to using DEA for the assessment of high frequencies. To accomplish this goal three datasets were generated. The first dataset contains results of the measurement of the experimental lightweight structure used in this paper. The second dataset contains FEM results for a 
validated model of the experimental structure. The third dataset contains results of the DEA simulation using a model derived from the FEM model. These datasets were evaluated using the ECC, an energy-based correlation criterion, which is able to assess linear dependencies for large frequency ranges as the $50-5000 \mathrm{~Hz}$ range observed in this paper.

The results showed that the two used models for FEM and DEA respectively deliver sufficient results compared to measurement data. The FEM model for low and high frequencies while the DEA only for high frequencies as it is explicitly developed for this frequency range. An ECC evaluation of the results of the FEM and DEA models directly showed that from frequencies of about $800-1000 \mathrm{~Hz}$ the DEA can be used instead of FEM to assess the high-frequency range. This frequency range only applies for the experimental lightweight structure used in this paper.

In order to provide a means for the transfer of the results to structures of other dimensions a rule-ofthumb based on analytical plate equations was derived. This rule ensures a rough estimate to what extent a lower frequency limit might decrease or increase. The proof of this scaling rule by proper numerical evaluation could not be finished by submission deadline of this paper but will be subject of future publications.

The topology of the experimental structure used in this paper was prone to the current modelling limitations of the DEA. Thus, the FE mesh used for the DEA simulations had deviations to the mesh used in FEM simulations. Therefore, it is expected that results improve significantly for applications in which there are no differences of the FE mesh used in DEA and the respective experimental structure.

\section{REFERENCES}

1. Fahy, F., Gardonio, P. (2007). "Sound and Structural Vibration". Oxford, Elsevier Academic Press.

2. Le Bot, A. (2015). "Foundation of statistical energy analysis in vibro-acoustics", Oxford University Press.

3. Keller, J. B. (1978). "Rays, waves and asymptotics." Bulletin of the American Mathematical Society 84: 727-750.

4. Tanner, G. (2008). "Dynamical energy analysis - Determining wave energy distributions in vibroacoustical structures in the high-frequency regime." Journal of Sound and Vibration 320: 10231038.

5. Hartmann, T., Satoshi, M., Tanner, G., Chappell, D.J. (2019). "High-frequency structure- and airborne sound transmission for a tractor model using Dynamical Energy Analysis." Wave Motion 87: $132-150$.

6. Chappell, D. J., Tanner, G., Giani, S. (2012). "Boundary element dynamical energy analysis: A versatile method for solving two- or three-dimensional wave problems in the high frequency limit." Journal of Computational Physics 231: 6181-6191.

7. Biedermann, J., Winter, R., Norambuena, M., Böswald, M., Wandel, M. (2017). „Classification of the mid-frequency range based on spatial Fourier decomposition of operational deflection shapes". Proceedings of International Congress on Sound and Vibration 24.

8. Biedermann, J., Winter, R., Wandel, M. and Böswald, M. (2017). "Energy based correlation criteria in the mid-frequency range," Journal of Sound and Vibration. 400: 457-480.

9. Allemang, R. J. (2002). "The Modal Assurance Criterion - Twenty Years of Use and Abuse". International Modal Analysis Conference. 20: 397-405.

10. Ewins, D. J. (2000). "Model validation: Correlation for updating." Sadhana 25: 221-234.

11. Dewald, R. D. (2020). „Modellierung und Modellvalidierung einer rippenversteiften Platte für vibroakustische Fragestellungen“. Institut für Dynamik und Schwingungen, Leibniz Universität Hannover: 97.

12. Winter, R., et al. (2018). "Fully automated vibration measurements of aircraft fuselages in the mid-frequency range". ISMA, Leuven.

13. Soedel, W. (2004). "Vibrations of Shells and Plates". Marcel Dekker Inc., New York. 